\title{
The Influence of Different Acupuncture Manipulations on the Skin Temperature of an Acupoint
}

\author{
Tao Huang, 1 Xin Huang, ${ }^{1}$ Weibo Zhang, ${ }^{1}$ Shuyong Jia, ${ }^{1}$ \\ Xinnong Cheng, ${ }^{1}$ and Gerhard Litscher ${ }^{1,2}$ \\ ${ }^{1}$ Institute of Acupuncture and Moxibustion, China Academy of Chinese Medical Science, Beijing 100700, China \\ ${ }^{2}$ Stronach Research Unit for Complementary and Integrative Laser Medicine, Research Unit of Biomedical Engineering in \\ Anesthesia and Intensive Care Medicine, TCM Research Center Graz, Medical University of Graz, 8036 Graz, Austria
}

Correspondence should be addressed to Tao Huang; dr_huangtao@sina.com and

Gerhard Litscher; gerhard.litscher@medunigraz.at

Received 28 October 2012; Accepted 3 December 2012

Academic Editor: Yi-Hung Chen

Copyright (C) 2013 Tao Huang et al. This is an open access article distributed under the Creative Commons Attribution License, which permits unrestricted use, distribution, and reproduction in any medium, provided the original work is properly cited.

This study was performed to observe the influence of sham and different verum acupuncture manipulations on skin temperature of the stimulated acupoint in healthy volunteers. Thirty-seven healthy volunteers with a mean age of $25.4 \pm 2.2$ years were enrolled in the study. All volunteers had experienced acupuncture before. They received sham acupuncture and two different kinds of verum acupuncture stimulation (lifting-thrusting and twisting-rotating) on Zusanli (ST36). The skin temperature of ST36 was measured before acupuncture, after needle insertion, after needle manipulation, immediately after removal of the needle, and as further control 5 minutes after removal of the needle using a FLIR i7 infrared thermal camera. During the measurement, the needling sensations of volunteers were enquired and recorded. During the sham acupuncture stimulation, the skin temperature of ST36 decreased in the first 5 minutes, when the point was exposed, and then increased gradually. During verum acupuncture stimulations, the skin temperature increased continually and then decreased in the last phase. The increase in temperature caused by liftingthrusting stimulation was significantly higher than that of twisting-rotating manipulation, which may be related to the stimulation intensity.

\section{Introduction}

Already in ancient times, doctors realized that acupuncture can influence the skin temperature. This is already mentioned in the first Chinese medical book Miraculous Pivot, where it is stated that "the doctor could warm the body through stimulating foot Shaoyin and cool the body through stimulating foot Yangming channels." So, even at that time it was well known that the skin temperature is an indicator of the reinforcing or reducing acupuncture manipulations. In Plain Questions, it is also stated that coldness and heating are part of the indexes of the acupuncture treatment principles.

Around the time of the Jin-Yuan dynasty, the compound manipulations like directional supplementation and draining method (迎随补泻, yingsui buxie), heat-producing needling (烧山火, shao shan huo), and cool-producing needling (透天凉, tou tian liang) were developed to treat diseases aided by heat or coldness feeling after acupuncture manipulation stimulation. These complex manipulations, however, are rarely used in current acupuncture clinic routine; many researchers focus on simpler acupuncture needle manipulations in their experiments and achieve different results.

This experiment tries to observe the influence of Cheng's basic acupuncture manipulations-lifting-thrusting and twisting-rotating - on local acupoint skin temperature in a randomized study, which may lay a foundation for further research.

\section{Materials and Methods}

2.1. Selection of Study Participants. Through advertisements on the campuses, 37 healthy volunteers ( 30 female, 7 male) with a mean age of $25.4 \pm 2.2$ years (range: $20-35$ years) were enrolled among students from the Beijing University of 


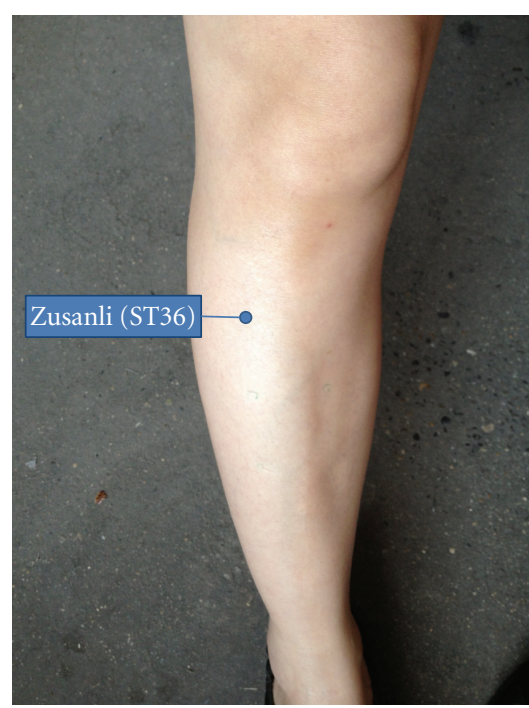

FIgURE 1: The location of the Zusanli acupoint (ST36).

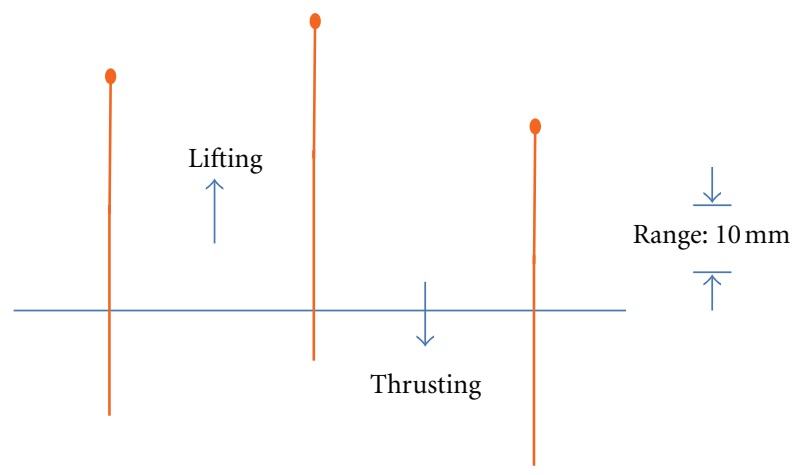

FIGURE 2: Lifting and thrusting acupuncture needle manipulation.

Traditional Chinese Medicine and Graduated School of the China Academy of Chinese Medical Sciences. All participants had received acupuncture before and gave informed consent. The experimental procedure was approved by the Ethics Committee of the Institute of Acupuncture and Moxibustion of China Academy of Chinese Medical Sciences.

2.2. Acupuncture. Each volunteer underwent three measurements (two different kinds of verum acupuncture, see below, and sham acupuncture, in randomized order) every other day. To avoid discrepancies in manipulation, all acupuncture operations were performed by the same medical practitioner. The volunteers lay down on the back and exposed the right lower leg, so the Zusanli (ST36) acupoint could be marked in accordance with a textbook on Acupuncture and Moxibustion [1] (see Figure 1). Verum acupuncture was performed according to Cheng [2].

Verum Acupuncture: Lifting and Thrusting Manipulation (提插, ticha). Acupuncture stimulation was done manually, using single-use acupuncture needles $(0.30 \times 40 \mathrm{~mm}$, Zhongyan Taihe brand, Suzhou, China). The doctor inserted

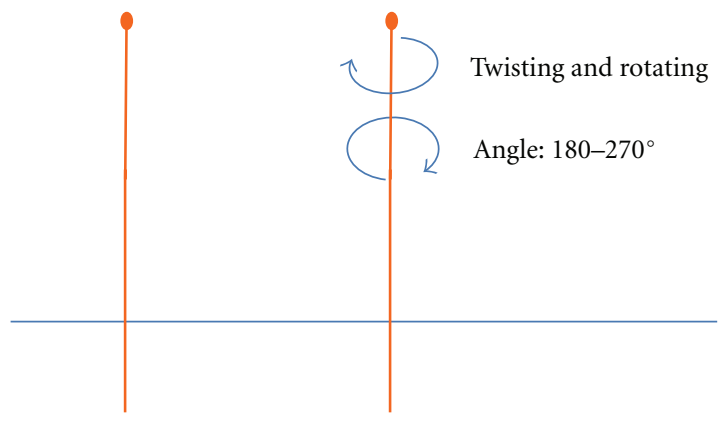

FIGURE 3: Twisting and rotating acupuncture needle manipulation.

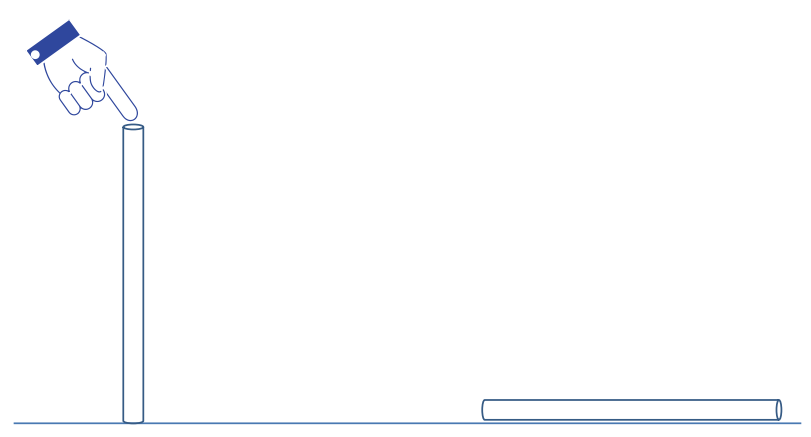

FIgURE 4: The operation of sham acupuncture.

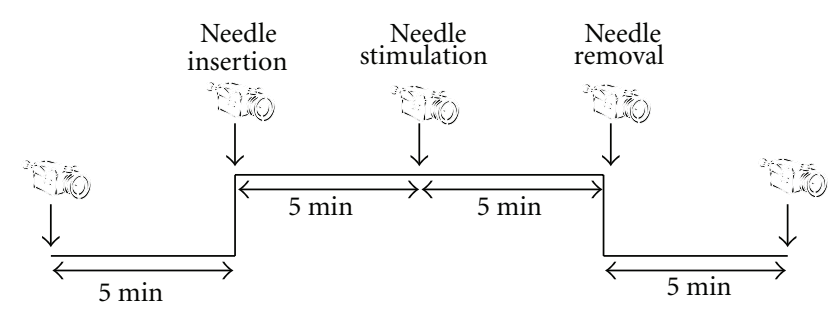

FIGURE 5: Measurement procedure.

the needle on ST36 through a tube to retain depth, until both the practitioner and the volunteer felt the qi arrival; then the insertion was stopped, and the needle remained in place. After 5 mins, the practitioner lifted and then thrust the needle evenly approximately $10 \mathrm{~mm}$ (see Figure 2), repeating this operation from 20 to 25 times in 20 seconds. The needle was left in place for 5 more mins and then removed.

Verum Acupuncture, Twisting and Rotating Manipulation (捻转, nianzhuan). Acupuncture stimulation was done manually, using the same single-use acupuncture needles as mentioned before. The doctor inserted the needle on ST36 through a tube to retain depth, until both the practitioner and the volunteer felt the qi arrival; then the insertion was stopped, and the needle remained in place (the same procedure as mentioned before). After 5 mins, the practitioner twisted the handle of the needle clockwise and counterclockwise evenly through $180^{\circ}$ to $270^{\circ}$ (see Figure 3), repeating this 

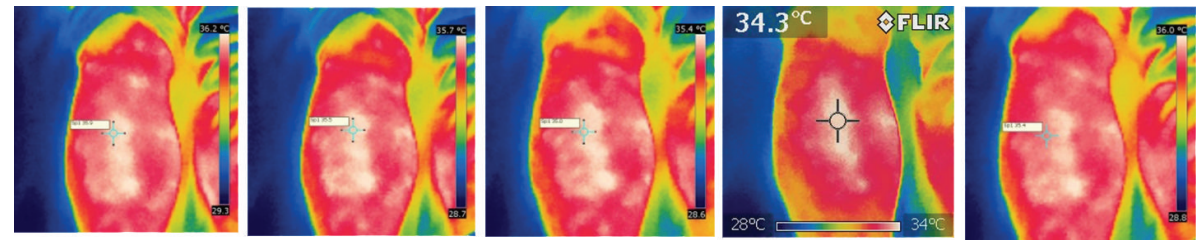

FIGURE 6: Temperature changes during the sham acupuncture procedure.
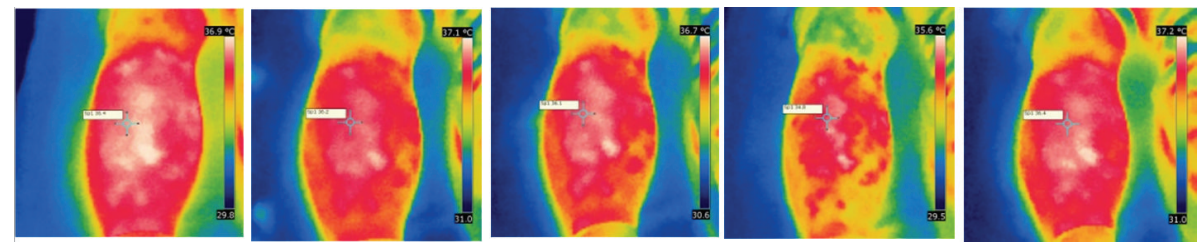

FIGURE 7: Temperature changes before, during, and after verum acupuncture-lifting-thrusting needle manipulation.

operation from 40 to 45 times in 20 seconds. The needle was left in place for 5 more mins and then removed.

Sham Acupuncture (Placebo). Sham acupuncture was performed using a single-use acupuncture needle tube (Zhongyan Taihe brand, Suzhou, China) which was tapped on ST36, but no needle was inserted and so the volunteers did not receive any stimulation (see Figure 4 ).

2.3. Measurement of Skin Temperature and Heart Rate. The temperature of the lab was kept at $26^{\circ} \mathrm{C}$, and the volunteers were asked to come into the room 5-10 mins ahead of schedule to adapt to the temperature. The skin temperature at ST36 was measured using a FLIR i7 (Flir Systems, Portland, OR, USA) thermographic camera 5 mins before acupuncture, immediately after needle insertion, after manipulation, immediately after removing the needle, and 5 mins after the needle removal (see Figure 5). During the whole experiment, the volunteers' heart rates (HR) were measured with three electrodes on standard positions of the chest, using a Medilog AR12 system (Huntleigh Healthcare, Cardiff, UK).

2.4. Needling Sensation. The volunteers were asked about their needling sensation after needle insertion and manipulation, respectively, were assessed by visual analogue scale (VAS). 0 means "no sensation at all," and 10 means "too much to bear."

2.5. Statistical Analysis. Paired $t$-test was used to compare the temperature changes between the different manipulations, with $P<0.05$ denoted as significant.

\section{Results}

3.1. Changes of HR. Sham as well as verum acupuncture caused changes in the volunteers' HR. After the $5 \mathrm{~min}$ phase of rest before acupuncture, HR decreased significantly. After verum acupuncture, needle manipulation, the liftingthrusting as well as the twisting-rotating stimulation induced a significant increase in HR (compared to the phase of needle insertion), whereas during the sham procedure HR continued to decrease (cf. Table 1).

3.2. Skin Temperature. After needle insertion, the skin temperature at ST36 decreased insignificantly following the sham procedure, but increased significantly in the two verum procedures. After manipulations, the temperature increase caused by lifting-thrusting stimulation was higher than that caused by sham acupuncture (see Figures 6, 7, and 8).

Table 2 shows the mean and standard deviation of the temperature values of all 37 volunteers.

3.3. Needling Sensations during Verum Acupuncture. All subjects could tell sham from verum acupuncture, but could not recognize the lifting-thrusting or twisting-rotating manipulations. After puncturing ST36, 71\% of the subjects felt distension, $36 \%$ felt sourness, $16 \%$ felt pricking, and $10 \%$ felt numbness spreading. There was of course no statistical difference in the intensity of the needling sensation when the needle was inserted, but the intensity caused by liftingthrusting stimulation was significantly higher than that of twisting-rotating manipulation (Table 3).

\section{Discussion}

Previous studies investigating acupuncture and skin temperature used compound reinforcing and reducing techniques to observe changes in skin temperature of the area along the meridian after puncturing the acupoint. A study by $\mathrm{Li}$ et al. showed that the skin temperature of acupoints changed according to the frequency of rotating acupuncture stimulation on ST36 [3]. A similar study from Tianjin in China showed that the skin temperature of the abdomen could be increased by reinforcing technique and decreased by reducing acupuncture stimulation technique on ST36 [4]. An experiment by Wang et al. in Shanghai showed that after twisting-rotating acupuncture stimulation with an angle $\leq 360^{\circ}$ on the acupoint SJ5 (Waiguan), the temperature of the ipsilateral acupoint PC1 (Zhongchong) increased [5]. A 

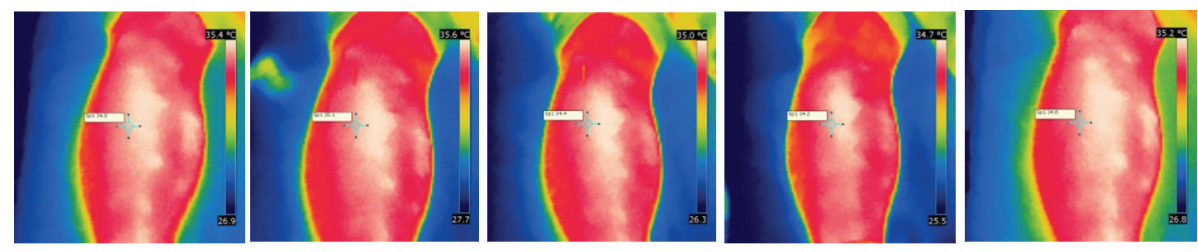

FIGURE 8: Temperature changes before, during, and after verum acupuncture-twisting-rotating needle manipulation.

TABLE 1: The changes of volunteers' HR (in $[1 / \mathrm{min}])$ during the experiment $\left({ }^{*} P<0.05\right)$.

\begin{tabular}{lccccc}
\hline & $\begin{array}{c}\text { Before } \\
\text { acupuncture }\end{array}$ & Needle insertion & $\begin{array}{c}\text { Needle } \\
\text { manipulation }\end{array}$ & Needle removal & After acupuncture \\
\hline Sham acupuncture & $71.03 \pm 9.04$ & $68.50 \pm 8.29^{*}$ & $68.15 \pm 7.84$ & $66.79 \pm 7.32$ & $67.82 \pm 8.04$ \\
Lifting-thrusting & $73.68 \pm 8.88$ & $69.84 \pm 9.93^{*}$ & $69.43 \pm 8.69$ & $69.95 \pm 9.71$ & $71.22 \pm 11.01^{*}$ \\
Twisting-rotating & $69.54 \pm 11.47$ & $65.27 \pm 12.28^{*}$ & $66.89 \pm 10.80$ & $67.32 \pm 9.63$ & $67.32 \pm 11.50^{*}$ \\
\hline
\end{tabular}

measurement in Hebei in China showed that after acupuncture stimulation on LI11 (Quchi), the skin temperature of the ipsilateral point LI1 (Shangyang) increased, too [6]. Similar to this experiment, Dong and Che could show that, after electroacupuncture stimulation on LI4 (Hegu), the temperature of LI4 increased significantly, decreasing slowly afterwards [7].

The lifting-thrusting and twisting-rotating needle manipulations described and investigated in this experiment come from one of the authors' (Professor X. Cheng) clinical experience. His very important results are summarized in Chinese Acupuncture and Moxibustion [2]. The clinical technique from Professor X. Cheng has influenced tens of thousands of acupuncturists all over the world. He emphasizes simple and direct clinical acupuncture techniques and uses liftingthrusting and twisting-rotating manipulations, stopping all operations when qi arrives. These techniques belong to middle and low dosages of stimulations. As his students, the authors adopt his ways in clinical routine and scientific experiments, which allows them to achieve good results $[8,9]$.

In one of these experiments [9], we used sham acupuncture as a control, considering it to represent the influence of environment, body position, and emotion. It was shown that, even without verum acupuncture stimulation, the skin temperature of the observed point changed significantly. This shows the necessity of a placebo control group in such investigations.

Skin is the only heat dissipation way of the human body; when the body energy metabolism and heat production increases, heat dissipation through the skin increases, too, and so the surface temperature rises [10]. There are three ways of skin heat dissipation: radiation, transmission, and evaporation. Thermal radiation is a procedure of living beings who emit heat in infrared rays to the surrounding environment; the higher the temperature compared to that of the surroundings, the higher the emission of infrared rays. Although we asked the volunteers to come to the lab early to adapt to the temperature, in the first 5 mins the temperature of the observational area decreased, following the exposure. The skin temperature of the acupoint then showed an increase caused by verum acupuncture with deqi arrival, whereas during the sham acupuncture procedure skin temperature continued to decrease. This shows that deqi acupuncture could increase skin temperature, which corresponds to the findings of one of our previous studies [9]. However, 5 minutes are quite short for determining baseline values, and, in further studies, this period should be expanded.

Both twisting-rotating (although not reaching the level of significance) and lifting-thrusting manipulations have been shown to make the skin temperature of the stimulated acupoint increase at first and decrease slowly later on. So, one can say that the lifting-thrusting method causes a stronger needling sensation and a stronger stimulation and induces a higher temperature at the stimulated acupoint. The stronger acupuncture stimulation may be related to the increase in blood perfusion $[11,12]$.

\section{Conclusion}

Compared to sham acupuncture, deqi acupuncture can be able to increase the skin temperature of the stimulated acupoint. The range of temperature increase caused by liftingthrusting stimulation is higher than that of the twistingrotating method. This may be connected with the stimulus intensity. Further research is needed to verify these findings.

\section{Conflict of Interests}

The authors declare that they have no conflict of interests.

\section{Acknowledgments}

The two corresponding authors T. Huang for China and Gerhard Litscher for Europe and all other authors are very thankful to Professor X. Cheng for his valuable help and agreement to be a coauthor of this paper. Their experiment is supported by the China National Science Foundation (81072760), the Returned personnel Foundation of MHRSS, and the Sino-Austrian projects (ZZ04007). The authors would like to thank Ms. Ingrid Gaischek, M.S., for her 
TABLE 2: Changes in skin temperature (in $\left[{ }^{\circ} \mathrm{C}\right]$ ) at ST36. The values in parentheses are the increases (decreases) of temperature with respect to the baseline values $\left({ }^{*} P<0.05\right)$.

\begin{tabular}{lccccc}
\hline & $\begin{array}{c}\text { Before } \\
\text { acupuncture }\end{array}$ & Needle insertion & $\begin{array}{c}\text { Needle } \\
\text { manipulation }\end{array}$ & Needle removal & $\begin{array}{c}\text { After } \\
\text { acupuncture }\end{array}$ \\
\hline Sham acupuncture & $34.87 \pm 1.14$ & $34.69 \pm 1.02$ & $35.18 \pm 0.67$ & $35.21 \pm 1.06$ & $35.23 \pm 0.78$ \\
& - & $(-0.18)$ & $(+0.31)$ & $(+0.34)$ & $(+0.36)$ \\
Lifting-thrusting & $34.53 \pm 1.01$ & $35.01 \pm 0.85$ & $35.37 \pm 0.79$ & $35.41 \pm 1.05$ & $35.35 \pm 1.13$ \\
& - & $(+0.48)$ & $(+0.84)^{*}$ & $(+0.88)^{*}$ & $(+0.82)^{*}$ \\
Twisting-rotating & $34.49 \pm 1.14$ & $34.64 \pm 1.38$ & $34.86 \pm 1.27$ & $34.96 \pm 1.09$ & $34.91 \pm 1.14$ \\
& - & $(+0.15)$ & $(+0.37)$ & $(+0.47)$ & $(+0.42)$ \\
\hline
\end{tabular}

TABLE 3: VAS scores expressing the intensity of the needling sensation caused by needle insertion and manipulations (mean $\pm \mathrm{SD}$ ).

\begin{tabular}{lcc}
\hline & $\begin{array}{c}\text { Needle } \\
\text { insertion }\end{array}$ & $\begin{array}{c}\text { Needle } \\
\text { manipulations }\end{array}$ \\
\hline Lifting-thrusting & $3.93 \pm 1.76$ & $7.13 \pm 1.90^{* *}$ \\
Twisting-rotating & $4.72 \pm 1.46$ & $4.91 \pm 2.32$ \\
\hline
\end{tabular}

valuable support in creating figures and preparing the paper. The investigations were supported by the two Austrian ministries of Science and Research and of Health (evidencebased high-tech acupuncture and integrative laser medicine for prevention and early interventions of chronic diseases), the Eurasia Pacific Uninet, and the Department of Science of the City of Graz. Gerhard Litscher is also a Visiting Professor of the Institute of Acupuncture and Moxibustion at China Academy of Chinese Medical Sciences, Beijing, China.

\section{References}

[1] X. M. Shi, New Century National High Education Textbook of Traditional Chinese Medicine. Acupuncture and Moxibustion, China Publishing House of Traditional Chinese Medicine, Beijing, China, 2007.

[2] X. N. Cheng, China Acupuncture and Moxibustion, Foreign Language Press, Beijing, China, 1999.

[3] T. Li, C. Y. Liu, X. K. Yan et al., "The influence for surface of stomach by different frequency of twisting-rotating acupuncture onST36," Journal of Clinical Acupuncture, vol. 27, no. 3, pp. 39-41, 2011.

[4] P. Li, W. Guan, F. Wang, B. Chen, and K. Zhu, "The influence of abdomen skin temperature caused by twisting-rotating reinforcing-reducing acupuncture manipulations on ST36 (Zusanli)," Tianjin Journal of Traditional Chinese Medicine, vol. 19, no. 4, pp. 51-54, 2002.

[5] C. H. Wang, Y. P. Wang, J. M. Xu, T. Y. Liu, and H. Y. Yang, "The influence of health people skin temperature by different quantity of stimulus twisting-rotating acupuncture manipulation," Shanghai Journal of Acupuncture and Moxibustion, vol. 26, no. 7, pp. 33-35, 2007.

[6] Y. J. Wang, H. Cai, and Z. Y. Hu, "The influence of health people skin temperature of points by different acupuncture manipulations," Hebei Journal of Traditional Chinese Medicine, vol. 16, no. 1, pp. 42-44, 2001.
[7] J. B. Dong and S. W. Che, The Infrared temperature measurement of acupuncture response on fixed point [M.S. thesis], Hebei University of Science and Technology, 2009.

[8] T. Huang, J. Kong, X. Huang, and Y. H. Xu, "Some misunderstandings of deqi phenomenon: from historic review to experimental study," Chinese Acupuncture \& Moxibustion, vol. 28, no. 2, pp. 105-109, 2008.

[9] T. Huang, R. H. Wang, W. B. Zhang et al., "Influence of transcutaneous $\mathrm{CO}_{2}$ emission between placebo and deqi acupuncture on acupoint," China Journal of Basic Medicine in Traditional Chinese Medicine, vol. 16, no. 12, pp. 1162-1166, 2010.

[10] H. X. Li, Physiology, Henan Medical University Publishing House, Zhengzhou, China, 1997.

[11] T. Huang, R. H. Wang, W. B. Zhang et al., "The influence of different methods of acupuncture on skin surface perfusion," Journal of Traditional Chinese Medicine, vol. 32, no. 1, pp. 4044, 2012.

[12] T. Huang, L. J. Yang, W. B. Zhang et al., "Observation of microvascular perfusion in the Hegu (LI4) acupoint area after deqi acupuncture at Quchi (LI11) acupoint using speckle laser blood flow scanning technology," Evidence-Based Complementary and Alternative Medicine, vol. 2012, Article ID 604590, 4 pages, 2012. 


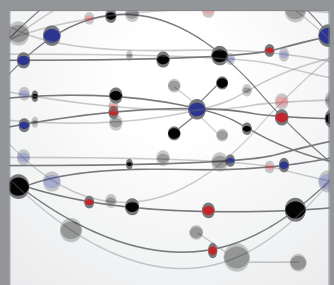

The Scientific World Journal
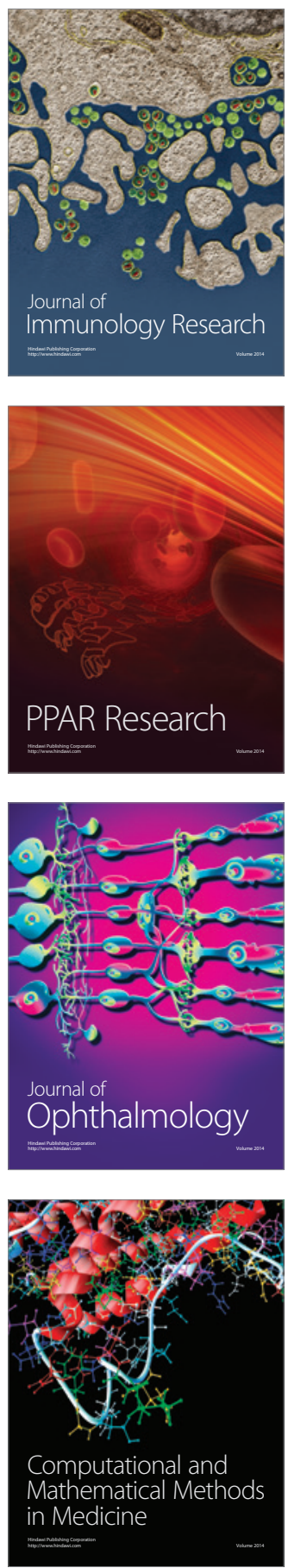

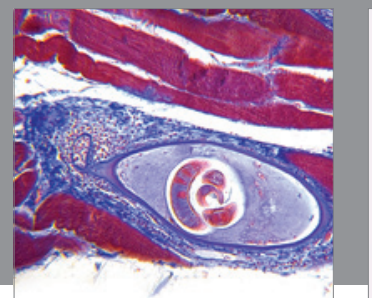

Gastroenterology

Research and Practice
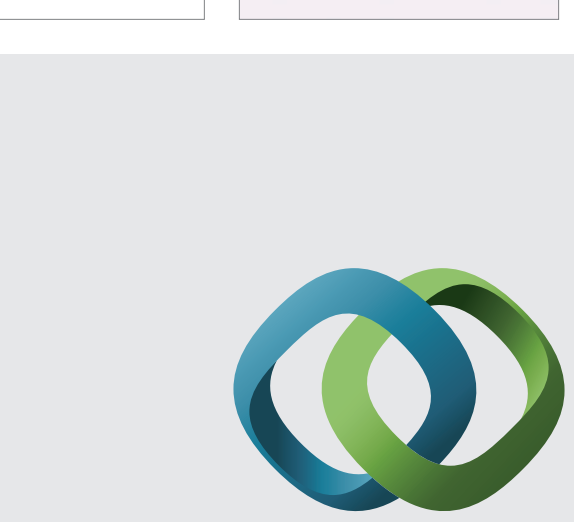

\section{Hindawi}

Submit your manuscripts at

http://www.hindawi.com
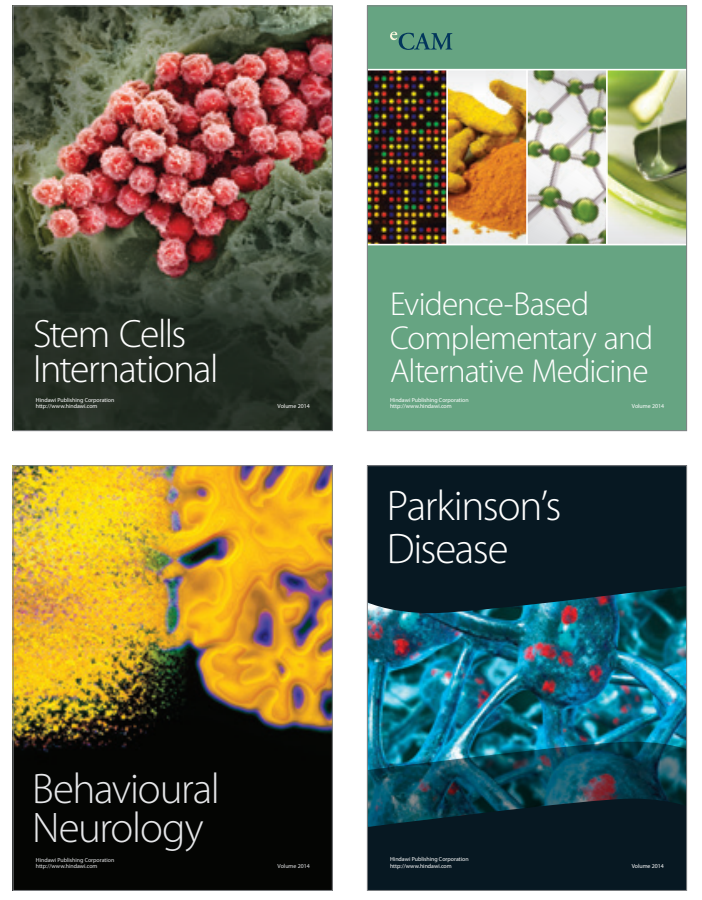
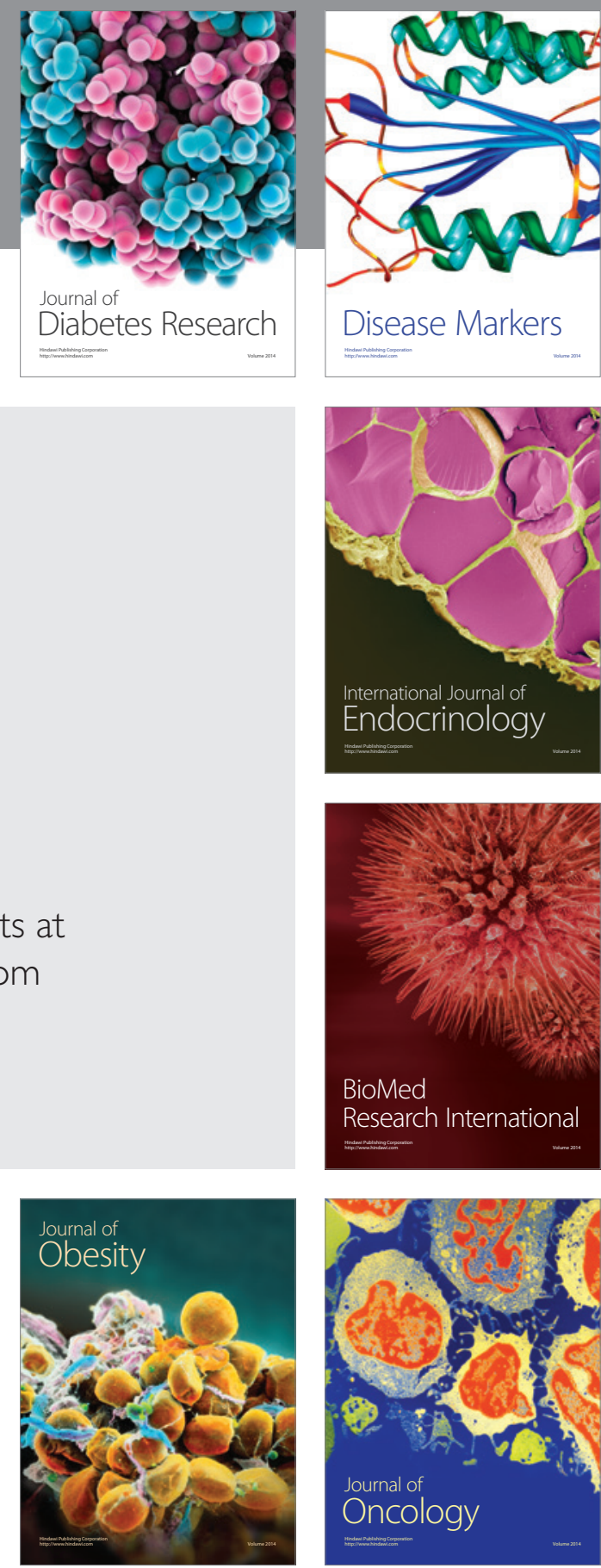

Disease Markers
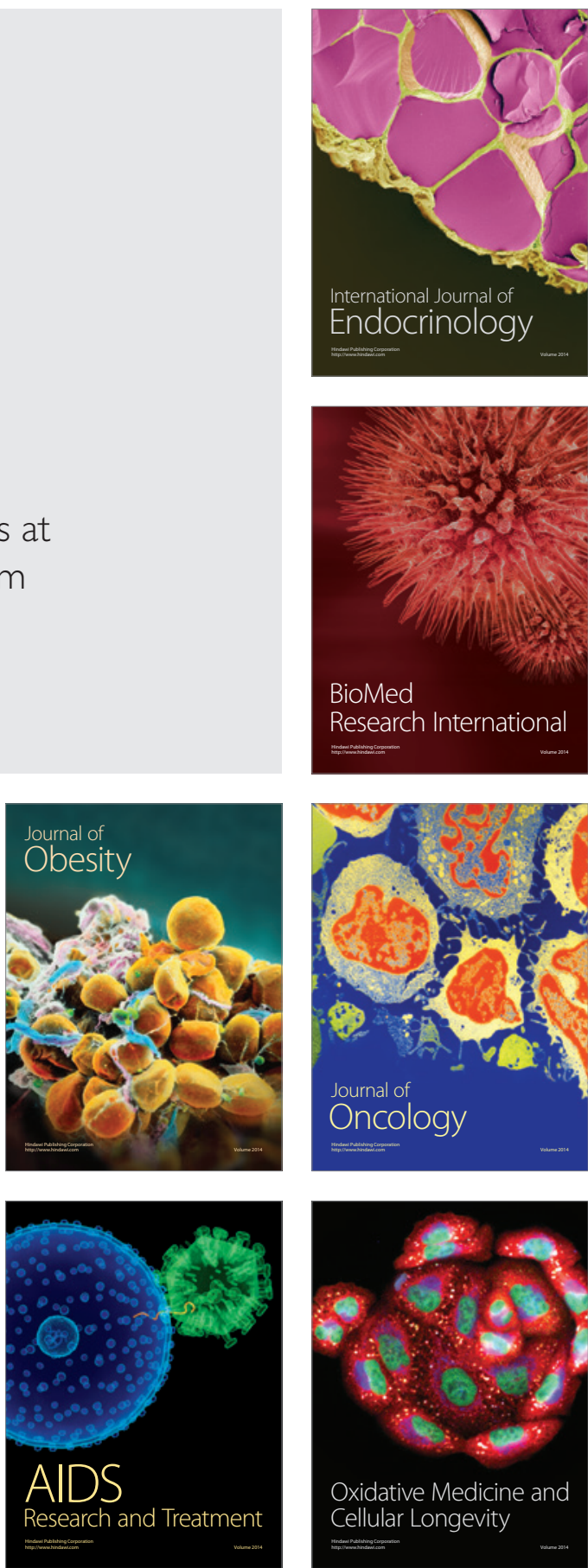